\title{
CORRECTIONS
}

\section{No more disease silos for sub-Saharan Africa}

An editorial slip changed the sense of a sentence in this Analysis by Patricio Marquez and Jill Farrington (BMJ 2012;345:e5812, doi:10.1136/bmj.e5812). The second sentence in the fifth paragraph should have noted that it is a lack of adequate (not "inadequate") social protection that will drive families and individuals further into poverty.

Cite this as: BMJ 2012;345:e5943

๑ BMJ Publishing Group Ltd 2012 